\title{
Anaplastic Lymphoma Kinase Regulates Internalization of the Dopamine D2 Receptor $\$$
}

\author{
Donghong He and (D)Amy W. Lasek \\ Center for Alcohol Research in Epigenetics and Department of Psychiatry, University of Illinois at Chicago, Chicago, Illinois
}

Received June 4, 2019; accepted November 11, 2019

\section{ABSTRACT}

The dopamine $\mathrm{D} 2$ receptor $(\mathrm{D} 2 \mathrm{R})$ is a $\mathrm{G}$ protein-coupled receptor (GPCR) expressed in regions of the brain that control motor function, cognition, and motivation. As a result, D2R is involved in the pathophysiology of disorders such as schizophrenia and drug addiction. Understanding the signaling pathways activated by $\mathrm{D} 2 \mathrm{R}$ is crucial to finding new therapeutic targets for these disorders. D2R stimulation by its agonist, dopamine, causes desensitization and internalization of the receptor. A previous study found that inhibitors of the receptor tyrosine kinase anaplastic lymphoma kinase (ALK) blocked D2R desensitization in neurons in the ventral tegmental area of the brain. In the present study, using a cell-based system, we investigated whether ALK regulates D2R internalization. The ALK inhibitor alectinib completely inhibited dopamine-induced D2R internalization. Since GPCRs can transactivate receptor tyrosine kinases, we also examined if D2R stimulation activated ALK signaling. ALK phosphorylation increased by almost 2-fold after dopamine treatment and ALK coimmunoprecipitated with D2R. To identify the signaling pathways downstream of ALK that might regulate D2R internalization, we used pharmacological inhibitors of proteins activated by ALK signaling. Protein kinase $\mathrm{C} \gamma$ was activated by dopamine in an ALK-dependent manner, and a protein kinase $\mathrm{C}$ inhibitor completely blocked dopamineinduced D2R internalization. Taken together, these results identify ALK as a receptor tyrosine kinase transactivated by D2R that promotes its internalization, possibly through activation of protein kinase C. ALK inhibitors could be useful in enhancing D2R signaling.

\section{SIGNIFICANCE STATEMENT}

Receptor internalization is a mechanism by which receptors are desensitized. In this study we found that agonist-induced internalization of the dopamine D2 receptor is regulated by the receptor tyrosine kinase ALK. ALK was also transactivated by and associated with dopamine D2 receptor. Dopamine activated protein kinase $\mathrm{C}$ in an ALK-dependent manner and a PKC inhibitor blocked dopamine D2 receptor internalization. These results indicate that ALK regulates dopamine D2 receptor trafficking, which has implications for psychiatric disorders involving dysregulated dopamine signaling.

\section{Introduction}

The neurotransmitter dopamine (DA) plays a critical role in Parkinson's disease and psychiatric disorders such as schizophrenia, obsessive-compulsive disorder, attention-deficit/ hyperactivity disorder, and drug addiction (Volkow and Morales, 2015; Howes et al., 2017). Understanding the neurophysiology of DA signaling is essential to developing new treatments for these brain disorders. DA activates DA receptors, which are G protein-coupled receptors (GPCRs) that are classified as D1like (D1R, D5R) or D2-like (D2R, D3R, and D4R) on the basis of

This work was supported by the National Institutes of Health, National Institute on Drug Abuse [Grant DA033429] and National Institute on Alcohol Abuse and Alcoholism [Grants AA020912; AA022538].

https://doi.org/10.1124/mol.119.117473. org. their coupling to the G-proteins $\mathrm{G} \alpha_{\mathrm{s}}$ or $\mathrm{G} \alpha_{\mathrm{i}}$, respectively. Like all GPCRs, the activity of DA receptors is tightly controlled by receptor desensitization as a cellular mechanism to terminate signaling after prolonged stimulation (Grady et al., 1997). Agonist-induced endocytosis is one mechanism of desensitizing receptors, by removing them from the cell surface and into the endocytic compartment. D1R and D2R differ in their endocytic mechanisms and fate once internalized (Vickery and von Zastrow, 1999; Bartlett et al., 2005). Whereas D2R undergoes both ligand-dependent and -independent constitutive endocytosis, very little D1R is internalized in the absence of ligand (Vickery and von Zastrow, 1999). In addition, D1R and D2R are localized to different endocytic vesicles after internalization (Vickery and von Zastrow, 1999). Finally, whereas agonist stimulation of D1R results in recycling back to the cell surface after internalization (Ariano et al., 1997; Vickery and von Zastrow, 1999; Bartlett

ABBREVIATIONS: ALK, anaplastic lymphoma kinase; BPA, biotin protection assay; BSA, bovine serum albumin; D1R, dopamine D1 receptor; D2R, dopamine D2 receptor; DA, dopamine; DAergic, dopaminergic; EGF, epidermal growth factor; EGFR, epidermal growth factor receptor; FBS, fetal bovine serum; Gö6976, 3-(13-Methyl-5-oxo-5,6,7,13-tetrahydro-12H-indolo[2,3-a]pyrrolo[3,4-c]carbazol-12-yl)propanenitrile; GPCR, G-protein-coupled receptor; HEK, human embryonic kidney; HRP, horseradish peroxidase; IP, immunoprecipitation; MAPK, mitogen-activated protein kinase; NVP-TAE684, 5-chloro-2-N-[2-methoxy-4-[4-(4-methylpiperazin-1-yl)piperidin-1-yl]phenyl]-4- $N$-(2-propan-2-ylsulfonylphenyl)pyrimidine-2,4-diamine; PBS, phosphate-buffered saline; PD98059, 2-(2-amino-3-methoxyphenyl)chromen-4-one; PDGFR, platelet-derived growth factor receptor; PKC, protein kinase C; PMA, phorbol 12-myristate 13-acetate; STAT3, signal transducer and activator of transcription 3; WB, Western blot; WP1066, (E)-3-(6-bromopyridin-2-yl)-2-cyano- $N$-[(1S)-1-phenylethyl]prop-2-enamide. 
et al., 2005), prolonged agonist stimulation causes D2R trafficking into lysosomes and subsequent receptor degradation (Bartlett et al., 2005).

In dopaminergic (DAergic) neurons of the ventral midbrain, $\mathrm{D} 2 \mathrm{R}$ functions as a regulatory autoreceptor that reduces excitability of DAergic neurons by activating $\mathrm{G}$ protein-gated inwardly rectifying potassium channels (Lacey et al., 1987). Bath application of DA onto brain slices containing the ventral tegmental area (VTA) initially inhibits the firing of DAergic neurons, but after prolonged exposure to DA, the inhibitory response is reduced and firing of DAergic neurons recovers, indicating that D2Rs have desensitized (Nimitvilai and Brodie, 2010). We previously found that inhibition of anaplastic lymphoma kinase (ALK), a receptor tyrosine kinase, blocked the recovery of inhibition by DA but not the initial DA-induced inhibition in VTA slices (Dutton et al., 2017), indicating that ALK plays a role in the desensitization of D2R.

We hypothesized that ALK regulates D2R desensitization by promoting endocytosis in response to prolonged DA stimulation, because ALK is internalized and degraded in lysosomes in response to agonist antibody treatment (Mazot et al., 2012), and because agonist stimulation of D2R transactivates receptor tyrosine kinases such as epidermal growth factor receptor (EGFR) and platelet-derived growth factor receptor (PDGFR) (Oak et al., 2001; Kotecha et al., 2002; Kim et al., 2004; Wang et al., 2005; Shioda et al., 2017). The purpose of this study was to determine if ALK: 1) plays a role in the internalization of D2R, 2) is transactivated by D2R stimulation, and 3) associates in a complex with D2R. We used human embryonic kidney (HEK) cells expressing FLAG-tagged versions of either D1R or D2R (Bartlett et al., 2005) to measure ALK-dependent internalization of D2R, transactivation of ALK by DA, and the association of ALK with D1R and D2R. Association of ALK with D2R and D2R-mediated activation of ALK was confirmed in a neuroblastoma cell line with endogenous expression of ALK and D2R.

ALK signals through many pathways, including the mitogenactivated protein kinase (MAPK), phosphatidylinositide 3 kinase (PI3K), signal transducer and activator of transcription 3 (STAT3), and protein kinase C (PKC) pathways (Palmer et al., 2009). Having demonstrated that ALK is transactivated by $\mathrm{D} 2 \mathrm{R}$ and regulates its endocytosis, we predicted that signaling downstream of ALK would promote internalization of D2R. The second goal of this study was to determine potential signaling pathways downstream of ALK that are involved in D2R internalization. Specifically, $\mathrm{PKC}$ is a prime candidate because it is known to regulate the internalization and desensitization of D2R (Namkung and Sibley, 2004; Morris et al., 2007; Thibault et al., 2011; Nimitvilai et al., 2012; Cho et al., 2013). We investigated whether inhibitors of PKC and other signaling pathways downstream of ALK regulate agonist-induced D2R internalization and found a selective effect of $\mathrm{PKC}$ inhibition on D2R internalization.

The results presented here indicate that ALK regulates D2R trafficking, possibly through a mechanism involving PKC. To our knowledge, this is the first demonstration that ALK is transactivated by a GPCR and regulates its internalization.

\section{Materials and Methods}

Chemicals, Antibodies, and Kits. Primary antibodies used were as follows: anti-FLAG M2 (cat. no. F1804, 1:1000; Sigma Aldrich),
anti-FLAG M2 affinity gel (cat. no. A2220; Sigma Aldrich), anti$\beta$-actin (clone AC-15, cat. no. A5441, 1:5000; Sigma Aldrich), antiALK (cat. no. 51-3900, 1:1000; Thermo Fisher Scientific), anti-pALK (phosphotyrosine 1278, clone D59G10; cat. no. 6941, 1:1000; Cell Signaling Technology), anti-pPKC $\gamma$ (phosphothreonine 514, clone EP2730Y, cat. no. ab109539, 1:1000; Abcam), anti-PKC $\gamma$ (cat. no. ab71558, 1:1000; Abcam), anti-PLC $\gamma 1$ (cat. no. 2822, 1:1000; Cell Signaling Technology), anti-pPLC $\gamma 1$ (phosphotyrosine 783, clone D6M9S, cat. no. 14008, 1:1000; Cell Signaling Technology), antidopamine D2 receptor (DRD2) (clone no. B-10, cat. no. sc-5303, 1: 200; Santa Cruz Biotechnology). Secondary antibodies used were goat anti-mouse IgG $(\mathrm{H}+\mathrm{L})$-horseradish peroxidase (HRP) conjugate (Bio-Rad), and goat anti-rabbit IgG $(\mathrm{H}+\mathrm{L})-\mathrm{HRP}$ conjugate (BioRad). Dopamine hydrochloride was purchased from Sigma-Aldrich, alectinib and WP1066 were purchased from Selleck Chemicals, Gö6976 was purchased from Tocris, PD98059 was purchased from Thermo Fisher Scientific, phorbol 12-myristate 13-acetate (PMA) was purchased from LC Laboratories, and glutathione, iodoacetamide, and Triton X-100 were purchased from Millipore Sigma. PNGase F was obtained from New England Biolabs. Pierce enhanced chemiluminescent (ECL) detection reagents, Halt protease inhibitor cocktail, EZ-Link Sulfo-NHS-SS-Biotinylation, and Pierce BCA protein assay kits were from Thermo Fisher Scientific. Vectastain ABC kit (HRP) was purchased from Vector Laboratories. $\mathrm{NaCl}, \mathrm{NaOH}, \mathrm{KCl}, \mathrm{CaCl}_{2}$, Tris, phosphate-buffered saline (PBS), Tween-20, and formaldehyde were purchased from Thermo Fisher Scientific. Cell Lysis Buffer was purchased from Cell Signaling Technology and nitrocellulose was from Bio-Rad.

Cell Culture. HEK-293FT cells (cat. no. R70007; ThermoFisher Scientific) and HEK cells stably expressing N-terminal FLAGtagged D1R and D2R-long isoform (kindly provided by Dr. Jennifer L. Whistler) were grown at $37^{\circ} \mathrm{C}$ with $5 \% \mathrm{CO}_{2}$ in Dulbecco's Modified Eagle's Medium (DMEM; Thermo Fisher Scientific) supplemented with $10 \%$ fetal bovine serum (FBS; Atlanta Biologicals). IMR-32 cells (CCL-127; American Type Culture Collection) were grown at $37^{\circ} \mathrm{C}$ and $5 \% \mathrm{CO}_{2}$ in Eagle's Minimum Essential Medium (Thermo Fisher Scientific) containing $10 \%$ FBS. Cells were cultured to 80\%-90\% confluency and starved in serum-free medium for 3 hours prior to treatments. For experiments with PKC $\gamma$ and control siRNAs [ON-TARGETplus Human PRKCG (5582) siRNA-SMARTpool and ON-TARGETplus Non-targeting Pool; Dharmacon, Inc.], cells were transfected with $100 \mathrm{nM}$ siRNA using Lipofectamine 2000 transfection reagent (Thermo Fisher) according to manufacturer's instructions. Cells were used for experiments 72 hours after transfection.

Biotin Protection Assay. HEK cells expressing D1R-FLAG or D2R-FLAG were grown to $80 \%-90 \%$ confluency in 10-cm plates. Cells were incubated with inhibitors (200 nM alectinib, $20 \mu \mathrm{M}$ Gö6976, $50 \mu \mathrm{M}$ PD98059, and $5 \mu \mathrm{M}$ WP1066) or $10 \mu \mathrm{M}$ PMA for 1 hour prior to treatment with DA. Cells were washed with cold PBS and then incubated with $3 \mu \mathrm{g} / \mathrm{ml}$ disulfide-cleavable biotin for 30 minutes at $4^{\circ} \mathrm{C}$. After incubation with biotin, cells were washed with PBS and placed in $37^{\circ} \mathrm{C}$ DMEM for 15 minutes before treatment with $10 \mu \mathrm{M}$ DA (or no treatment) for 60 minutes at $37^{\circ} \mathrm{C}$. Separate plates with total cell surface biotin (total) and biotin stripped from the cell surface (strip) remained at $4^{\circ} \mathrm{C}$ while experimental plates were treated with DA. After treatment, plates were washed with PBS, and remaining cell surface biotinylated receptors were stripped of biotin (except the "total" plate) using $50 \mathrm{mM}$ glutathione, $75 \mathrm{mM} \mathrm{NaCl}, 75 \mathrm{mM} \mathrm{NaOH}$, and $10 \% \mathrm{FBS}$ at $4^{\circ} \mathrm{C}$ for 30 minutes. Reactions were quenched with $50 \mathrm{mM}$ iodoacetamide, $10 \% \mathrm{BSA}$, and Tris- $\mathrm{HCl}$ buffer ( $\mathrm{pH} 7.4$ ), then lysed in $1 \mathrm{mg} / \mathrm{ml}$ iodoacetamide, $0.1 \%$ Triton X-100, $150 \mathrm{mM} \mathrm{NaCl}$,

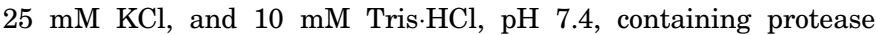
inhibitors. Lysates were cleared of insoluble material by centrifugation $\left(16,000 \mathrm{~g}\right.$ for 10 minutes at $\left.4^{\circ} \mathrm{C}\right)$. Cleared lysates $(1 \mathrm{mg}$, determined using BCA kit) were immunoprecipitated with $20 \mu \mathrm{l}$ of anti-FLAG M2 affinity gel, washed three times with immunoprecipitation (IP) buffer (same buffer as above without iodoacetamide), and then treated with PNGase 

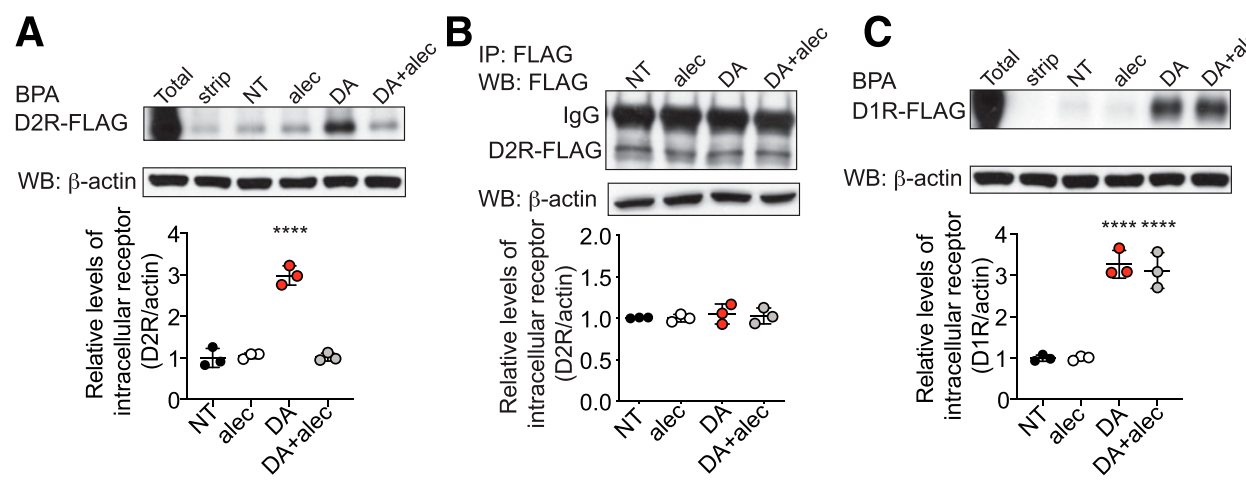

Fig. 1. D2R internalization is blocked by the ALK inhibitor alectinib. (A) Biotin protection assay of HEK cells expressing FLAG-tagged D2R (D2R-FLAG) after 1 hour of dopamine treatment showing internalization of D2R-FLAG and blockade by pretreatment with alectinib (alec). Top blot: representative streptavidin blot of internalized biotinylated D2R-FLAG. Bottom blot: representative Western blot (WB) of total cell lysates probed with an antibody to $\beta$-actin. Labels on blots: total, biotinylated cell surface D2R-FLAG prior to incubation with DA (positive control); strip, biotinylated D2R-FLAG prior to incubation with DA and after stripping biotin from cell surface (negative control); NT, no treatment; alec, $200 \mathrm{nM}$ alectinib treatment; DA, $10 \mu \mathrm{M}$ dopamine treatment. Graph shows relative levels of D2R/actin quantified with NIH ImageJ software. ****P<0.0001 by post-hoc Tukey's test after oneway ANOVA when comparing DA treatment with NT, alec, and DA+alec. (B) Immunoprecipitation and WB showing that total levels of D2R-FLAG after 60-minute treatment of cells with DA and alectinib are not altered. Top blot: representative WB of cell lysates that were immunoprecipitated with FLAG antibody and probed with FLAG antibody. Bottom blot: representative blot of total cell lysates that were probed with antibody to $\beta$-actin. Graph shows quantified levels of D2R/ $\beta$-actin. IgG, immunoglobulin heavy chain. (C) BPA of HEK cells expressing FLAG-tagged D1R (D1R-FLAG) showing that alec does not affect the internalization of D1R. Blots are labeled as in (A). ****P<0.0001 by post-hoc Tukey's test after one-way ANOVA, comparing DA and DA+alec treatment with NT and alec. All data are plotted as the mean \pm S.D. of three independent experiments.

F for 1 hour at $37^{\circ} \mathrm{C}$. Sample loading buffer containing sodium dodecyl sulfate and $\beta$-mercaptoethanol was added (Bio-Rad) and samples were resolved on Tris-glycine gels (Novex 4\%-12\% gradient; Thermo Fisher Scientific) under denaturing conditions with sodium dodecyl sulfatecontaining running buffer. Resolved proteins were transferred to nitrocellulose membranes and visualized with Vectastain $\mathrm{ABC}$ kit (HRP).

Western Blots. Cells were lysed in 1X Cell Lysis Buffer $(20 \mathrm{mM}$ Tris-HCl, pH 7.5, 150 mM NaCl, 1 mM EDTA, 1 mM EGTA, $1 \%$ Triton $\mathrm{X}-100,2.5 \mathrm{mM}$ sodium pyrophosphate, $1 \mathrm{mM} \beta$-glycerophosphate, $1 \mathrm{mM} \mathrm{Na} \mathrm{VO}_{4}$, and $1 \mu \mathrm{g} / \mathrm{ml}$ leupeptin; Cell Signaling Technology) supplemented with Halt protease inhibitor cocktail. Lysates were clarified by centrifugation at $16,000 \mathrm{~g}$ for 5 minutes at $4^{\circ} \mathrm{C}$. Protein concentrations were determined using the BCA protein assay kit and equal amounts of protein $(20-25 \mu \mathrm{g})$ were subjected to gel electrophoresis on Tris-glycine gels $(10 \%$ or $4 \%-12 \%)$ and transferred to nitrocellulose membranes. Membranes were blocked with 5\% BSA or $5 \%$ milk in TBST ( $25 \mathrm{mM}$ Tris-HCl, $137 \mathrm{mM} \mathrm{NaCl}$ and $0.1 \%$ Tween 20) and incubated with primary antibodies overnight at $4^{\circ} \mathrm{C}$. The membranes were then incubated with HRP-conjugated secondary antibody (1:2000) at room temperature and developed using enhanced chemiluminescent detection reagents. Films were developed using a Konica SRX-101A film processer. Films were scanned and densitometry performed using NIH ImageJ software.

Immunoprecipitation. HEK cells expressing D1R-FLAG or D2R-FLAG were washed twice with PBS and lysates were prepared in lysis buffer as described above for Western blots. Protein concentrations were determined with a BCA protein assay kit. Equal amounts of protein $(500-800 \mu \mathrm{g})$ were incubated with $20 \mu \mathrm{l}$ antiFlag M2 affinity gel overnight at $4^{\circ} \mathrm{C}$. Samples were centrifuged at $5000 \mathrm{~g}$ for 5 minutes and washed three times with ice-cold PBS. Protein-agarose complexes were dissociated by boiling for 5 minutes in sample loading buffer. Samples were run on Tris-glycine gels and Western blotted as described above. For IMR-32 cells, IP and Western blotting were performed as described above, except that lysates were immunoprecipitated with antibody to D2R and protein $\mathrm{A} / \mathrm{G}$ agarose beads (Santa Cruz Biotechnology).

Statistical Analysis. Statistical analysis was done using GraphPad Prism software (version 8). Data in Figs. 1, 3, 4, D-F, and 5 were analyzed by one-way ANOVA followed by post-hoc Tukey's multiple comparisons test. Data in Figs. 2 and 4, B and C, were analyzed by two-way ANOVA followed by post-hoc Tukey's multiple comparisons testing. Data were accepted as statistically significant with a $P$-value $<0.05$.

\section{Results}

D2R Internalization Is Blocked by the ALK Inhibitor Alectinib. To determine if ALK is involved in agonistinduced $\mathrm{D} 2 \mathrm{R}$ internalization, we performed a biotin protection assay (BPA), in which cell surface receptors are labeled with disulfide-cleavable biotin and internalized receptors are "protected" from biotin cleavage by membrane-impermeant glutathione. HEK cells expressing N-terminal FLAG-tagged D1R (D1R-FLAG) or D2R (D2R-FLAG) (Bartlett et al., 2005) were labeled with biotin after a preincubation with the selective ALK inhibitor alectinib. Cells were then treated with DA for 1 hour to induce receptor internalization. Remaining cell surface receptors were stripped of biotin using glutathione buffer and cell lysates were immunoprecipitated with anti-FLAG M2 affinity gel and analyzed by streptavidin overlay to measure intracellular biotinylated receptors. As expected, treatment with DA promoted a 3 -fold increase in internalized D2R-FLAG. Pretreatment with alectinib, however, abolished DA-induced internalization (Fig. 1A) without changing total D2R-FLAG levels (Fig. 1B). Likewise, treatment with the D2R agonist quinpirole promoted D2R-FLAG internalization, which was reduced by treatment with alectinib (Supplemental Fig. 1). DA treatment also induced a 3.3fold increase in internalized D1R-FLAG; however, alectinib had no effect on DA-induced D1R-FLAG internalization (Fig. 1C). These results suggest that ALK is involved in the internalization of D2R but not D1R.

ALK Associates with D2R and Is Activated by DA Treatment. On the basis of the observation that ALK regulates D2R internalization, we hypothesized that ALK may be either directly or indirectly associated with D2R. To test this, we performed an IP experiment in HEK cells expressing D1RFLAG or D2R-FLAG. Cells were treated with DA for 0,15 , or 30 minutes. Lysates were then immunoprecipitated with FLAG 
A

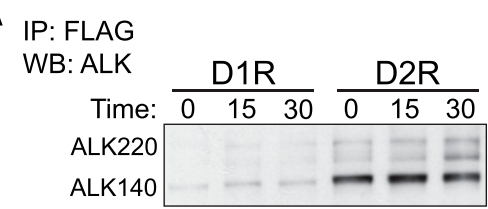

IP: FLAG

WB: FLAG

D2/D1R FLAG

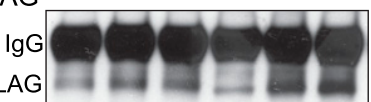

B

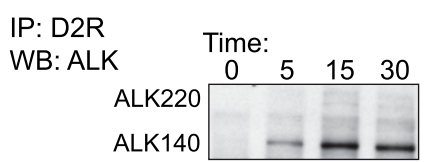

IP: D2R

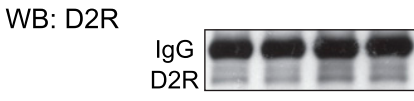

WB: ALK

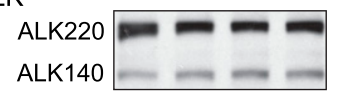

IMR-32

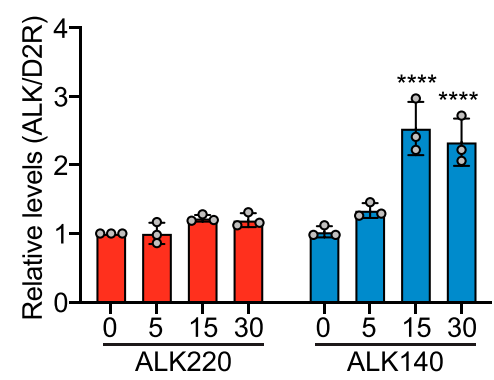

C
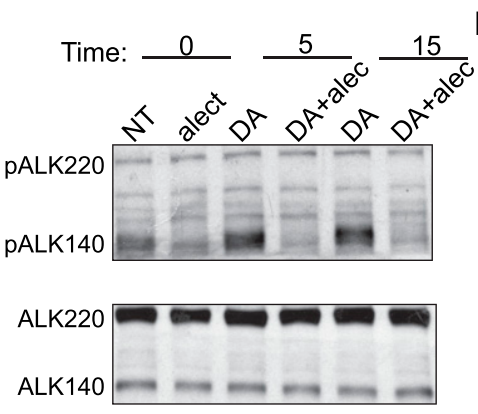

HEK

ALK220 ALK140 ALK220 ALK140

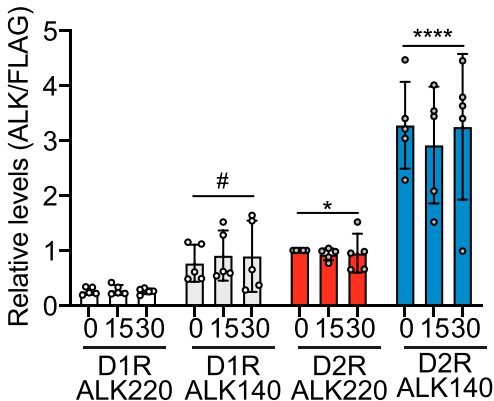

Fig. 2. ALK associates with D2R and is activated by DA treatment. (A) ALK 220 and $140-\mathrm{kDa}$ isoforms associated with D2R-FLAG. HEK cells expressing D1R-FLAG or D2R-FLAG were incubated with $10 \mu \mathrm{M}$ DA for 0,15 , or 30 minutes. Cell lysates were immunoprecipitated (IP) with FLAG antibody and WB with ALK (top blot) or FLAG (bottom blot) antibody. Graph shows intensity of ALK/FLAG bands (relative to untreated ALK 220/ FLAG) on blots plotted as mean \pm S.D. from five independent experiments. Post-hoc Tukey's test (collapsed across time): $* * * * P<0.0001$ when comparing ALK 140 in D2R-FLAG to D1R-FLAG cells and ALK 220 to ALK 140 in D2R-FLAG cells. ${ }^{*} P<0.05$ when comparing ALK 220 in D2R-FLAG vs. D1R-FLAG cells. ${ }^{\#} P<0.05$ when comparing ALK 220 to ALK 140 in D1RFLAG cells. (B) ALK 140 associated with D2R. IMR-32 cells were treated with DA for the indicated times. Lysates were immunoprecipitated with D2R antibody and WB with either ALK (top blot) or D2R antibody (middle blot). Bottom blot shows cell lysates probed with antibody to total ALK. Post-hoc Tukey's test: **** $P<$ 0.0001 when comparing ALK 140 at 0 minutes to ALK 140 at either 15 or 30 minutes. Graph shows mean \pm S.D. from three independent experiments. (C) DA treatment increased phosphorylation of ALK 140. IMR-32 cells were incubated with DA \pm alec for the indicated time and lysates were probed by WB with antibody to phosphorylated ALK (pALK) or total ALK protein. There was a significant increase in pALK 140 after DA treatment, which was blocked by treatment with alec. Post-hoc Tukey's test: $* * * * P<0.0001$ when compared with NT $(0$ minute) within each time point; $* * * P<$ 0.001 when comparing pALK 140 between alec-treated vs. non-alec-treated collapsed across time points. Graph shows mean \pm S.D. from three independent experiments. antibody followed by Western blotting with antibody to ALK. In cells expressing D2R-FLAG, bands for the 220- and 140-kDa isoforms of ALK (ALK 220 and ALK 140) were evident after FLAG IP (Fig. 2A). Interestingly, ALK 140 showed greater association with D2R than full-length ALK 220 (approx. 3-fold increase). In contrast, very low levels of ALK 220 and ALK 140 were observed on Western blots after FLAG IP in cells expressing D1R-FLAG. DA treatment did not affect the amount of ALK IP with D2R-FLAG.

We confirmed the association of ALK with D2R in another cell line, IMR-32, which is a human neuroblastoma cell line that expresses wild-type ALK (Janoueix-Lerosey et al., 2008) and D2R (He and Lasek, unpublished data). IMR-32 cells were treated with DA for $0,5,15$, or 30 minutes, and then lysates were immunoprecipitated with antibody to D2R followed by Western blots with ALK antibody. Similar to our findings in HEK cells expressing D2R-FLAG, ALK 220 and ALK 140 were associated with endogenous D2R, with more ALK 140 associated with D2R than ALK 220 (Fig. 2B). Treatment of IMR-32 cells with DA increased the association of ALK with D2R (2.5fold increase in ALK 140 after 15 minutes of DA treatment), in contrast to what was observed in HEK cells expressing D2RFLAG. The specificity of the ALK-D2R interaction was confirmed by incubating cell lysates with protein $\mathrm{A} / \mathrm{G}$ agarose beads without D2R antibody. ALK was not associated with $\mathrm{D} 2 \mathrm{R}$ in this negative control experiment (Supplemental Fig. 2). Together, these results suggest that ALK can associate in a complex with $\mathrm{D} 2 \mathrm{R}$.

Activation of $\mathrm{D} 2 \mathrm{R}$ is known to transactivate receptor tyrosine kinases (Oak et al., 2001; Kotecha et al., 2002; Kim et al., 2004; Wang et al., 2005; Shioda et al., 2017). The association of ALK with D2R suggests that agonist-induced D2R activation might transactivate ALK. IMR-32 cells were treated with DA for 0,5 , or 15 minutes and cell lysates were probed with antibodies to ALK and phosphorylated ALK (pALK, Y1278) by Western blot. DA treatment induced phosphorylation specifically of ALK 140 (1.7-fold increase) but not of ALK 220, an effect that was completely blocked by pretreatment with the ALK inhibitor alectinib (Fig. 2C). pALK 140 levels were also increased by 2 -fold at 5 minutes and 1.5fold at 15 minutes after treatment of IMR-32 cells with the D2R agonist quinpirole (Supplemental Fig. 3). These results indicate that in addition to associating with the D2R, ALK 140 is transactivated by $\mathrm{D} 2 \mathrm{R}$ in response to $\mathrm{D} 2 \mathrm{R}$ agonists.

PKC Inhibition Blocks Internalization of D2R. D2R phosphorylation by PKC promotes D2R internalization and desensitization (Namkung and Sibley, 2004; Morris et al., 2007; Thibault et al., 2011; Cho et al., 2013). Since ALK can 


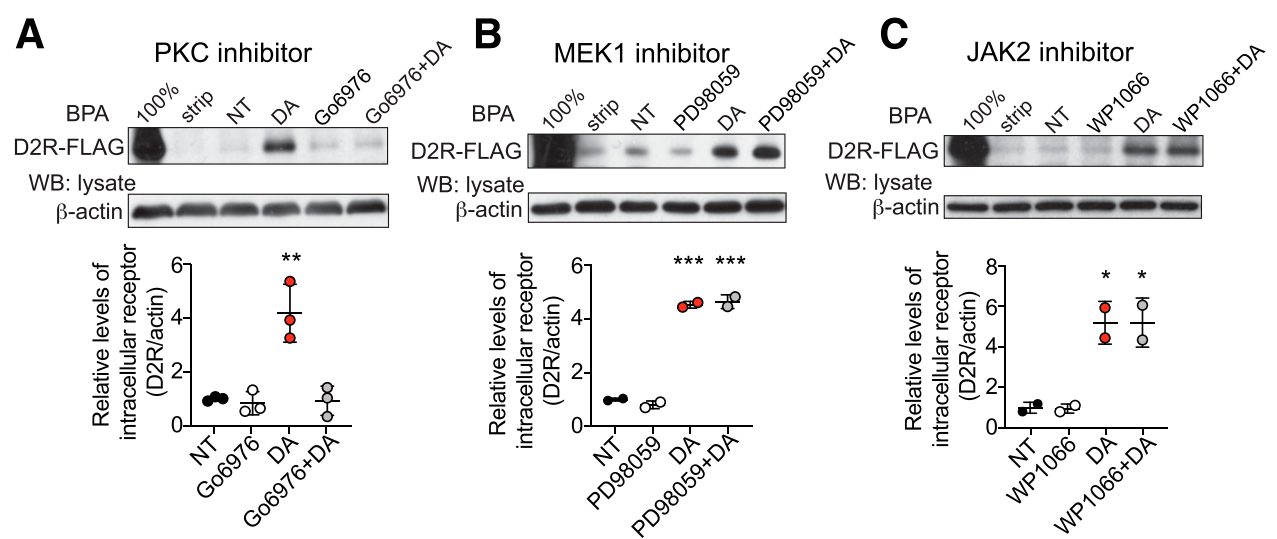

Fig. 3. PKC inhibition blocks D2R internalization. BPA of HEK cells expressing D2R-FLAG after treatment of 1 hour with $10 \mu \mathrm{M} D A \pm$ inhibitors to (A) PKC (Gö6976, $20 \mu \mathrm{M})$, (B), MEK1 (PD98059, $50 \mu \mathrm{M}$ ), and (C) JAK2 (WP1066, $5 \mu \mathrm{M}$ ). For each experiment, the top blot is a representative streptavidin blot of internalized biotinylated D2R-FLAG and the bottom blot is the corresponding blot of total cell lysates probed with $\beta$-actin antibody. Labels on blots: total, biotinylated cell surface D2R-FLAG prior to incubation with DA (positive control); strip, biotinylated D2R-FLAG prior to incubation with DA and after stripping biotin from cell surface (negative control); NT, no treatment. Graphs show the mean \pm S.D. of three (A) or two (B and C) independent experiments. ${ }^{* * P}<0.01$ when compared with NT, Gö6976, and Gö6976+DA (A), ***P<0.001 when compared with NT and PD98059 (B), and $* P<0.05$ when compared with NT and WP1066 by post-hoc Tukey's test after one-way ANOVA.

activate PKC through phospholipase C (PLC $\gamma 1)$ (Crockett et al., 2004; Van den Eynden et al., 2018), we postulated that this might be a potential mechanism by which ALK promotes D2R internalization. To first establish whether inhibition of PKC would block DA-induced internalization of D2R, we performed BPA in HEK cells expressing D2RFLAG. Cells were pretreated with Gö6976, an inhibitor of the conventional ( $\mathrm{Ca}^{+}$-dependent) PKC isoforms, and then treated with DA for 1 hour to induce endocytosis of D2R-FLAG. DA treatment resulted in internalization of D2R-FLAG, while pretreatment with Gö6976 abolished internalization (Fig. 3A). To determine if inhibition of other signaling proteins downstream of ALK altered the internalization of D2R, we pretreated cells with an inhibitor of MEK1 (PD98059), a member of the ERK/MAPK pathway. PD98059 did not affect DA-induced internalization of D2RFLAG (Fig. 3B). The STAT3 signaling pathway is activated by ALK (Sattu et al., 2013). Because Gö6976 inhibits JAK2, a kinase upstream of STAT3, it was possible that the ability of Gö6976 to block DA-induced internalization of D2R resulted from inhibition of JAK2, rather than PKC. To rule out this possibility, we treated cells with the JAK2 inhibitor WP1066 and measured internalization of D2R. WP1066 had no effect on internalization of D2R-FLAG (Fig. 3C). These results indicate that conventional PKCs are involved in the DA-induced endocytosis of D2R, whereas the ERK/MAPK and JAK2/STAT3 signaling pathways likely do not play a role in trafficking of $\mathrm{D} 2 \mathrm{R}$.

DA Induces Phosphorylation of PKC $\gamma$ and PLC $\gamma 1$ in an ALK-Dependent Manner. We next investigated if treatment with DA could induce phosphorylation of PLC $\gamma 1$ ( $\mathrm{PLC} \gamma 1$ ), an upstream regulator of PKC, and various isoforms of conventional and novel PKCs (pPKC) in an ALKdependent manner, since PLC $\gamma 1$ interacts with ALK (Crockett et al., 2004). HEK cells expressing D2R-FLAG were treated with $\mathrm{DA} \pm$ alectinib for $0,5,15$, or 30 minutes and lysates were analyzed for levels of total and phosphorylated PKC $\alpha, \operatorname{PKC} \beta 1$, $\mathrm{PKC} \gamma, \mathrm{PKC} \theta$, and $\mathrm{PLC} \gamma 1$. DA rapidly and transiently increased $\operatorname{pPKC} \beta 1, \mathrm{pPKC} \theta, \mathrm{pPKC} \gamma$, and pPLC $\gamma 1$, but not $\mathrm{pPKC} \alpha$ (Fig. 4; Supplemental Fig. 4). Alectinib pretreatment attenuated DAinduced increases in $\mathrm{pPKC} \gamma, \mathrm{pPKC} \theta$, and $\mathrm{pPLC} \gamma 1$, indicating that DA-induced phosphorylation of these proteins was at least partially dependent on ALK activation. These results indicate that $\mathrm{DA}$ can activate $\operatorname{PLC} \gamma 1, \operatorname{PKC} \gamma$, and $\operatorname{PKC} \theta$ through ALK.

We next determined whether reducing levels of PKC $\gamma$ would alter DA-induced internalization of D2R. HEK cells expressing D2R-FLAG were transfected with either a control siRNA pool or a pool of siRNAs targeting PKC $\gamma$ and subjected to BPA 72 hours after transfection. PKC $\gamma$ protein was reduced in cells transfected with $\operatorname{PKC} \gamma$ siRNAs by approx. 50\% compared with cells transfected with the control siRNA. A corresponding approx. 50\% decrease in DA-induced internalization of D2R occurred in cells transfected with the PKC $\gamma$ siRNAs (Fig. 4, D-F). These data demonstrate that $\mathrm{PKC} \gamma$ contributes to DA-induced internalization of D2R in this heterologous system and suggests that DA-induced activation of PKC $\gamma$ through ALK may promote internalization of D2R.

PKC Activation by the Phorbol Ester PMA Eliminates the Ability of Alectinib to Block D2R Internalization. We hypothesized that the activation of PKC through a PLC- and ALK-dependent mechanism is probably involved in the agonist-induced D2R internalization. If ALK is upstream of PKC-mediated D2R endocytosis, then direct activation of PKC should eliminate the ability of alectinib to block internalization of the D2R. To test this, we performed a BPA with HEK cells expressing D2R-FLAG after treatment with DA, PMA, and alectinib. As demonstrated above, DA induced D2R-FLAG internalization (a 4.4-fold increase compared with untreated), whereas pretreatment with alectinib attenuated D2R-FLAG internalization (Fig. 5). Brief treatment with PMA in the absence of DA did not statistically significantly increase D2R-FLAG internalization (a 1.5-fold increase compared with untreated), nor did PMA alter DA-induced D2R-FLAG internalization (a 4.4fold increase compared with untreated). However, PMA blocked the ability of alectinib to inhibit DA-induced internalization of D2R (Fig. 5, a 4.4-fold increase in internalized receptor compared with untreated). Taken together, these results suggest that ALK activation might act upstream of PKC to promote D2R internalization. 

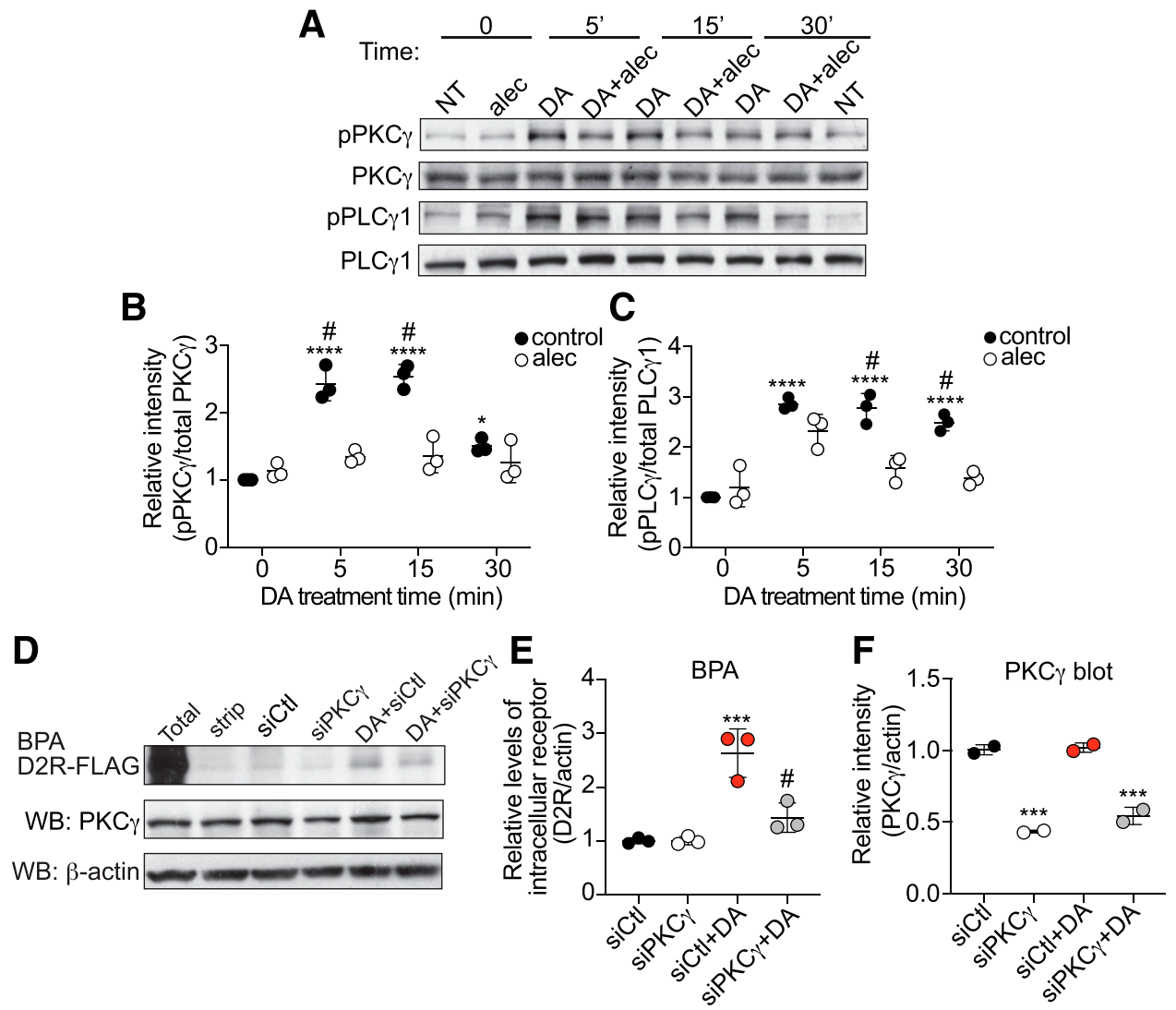

Fig. 4. DA induces PKC $\gamma$ and PLC $\gamma 1$ phosphorylation in an ALK-dependent manner. HEK cells expressing D2R-FLAG were treated with dopamine (DA, $10 \mu \mathrm{M}) \pm$ alectinib (alec, $200 \mathrm{nM}$ ) for 0,5 , 15, or 30 minutes. Cell lysates were prepared and subjected to Western blotting for phosphorylated PKC $\gamma$ (pPKC $\gamma$, T514), total PKC, phosphorylated PLC $\gamma 1$ (pPLC $\gamma 1$, Tyr783), and total PLC $\gamma 1$. (A) Representative western blots. NT, no treatment. (B) Quantification of relative band intensity of pPKC $\gamma / \mathrm{PKC} \gamma$ on Western blots. (C) Quantification of relative band intensity of pPLC $\gamma 1 / \mathrm{PLC} \gamma 1$ on western blots. All data are shown as the mean \pm S.D. of three independent experiments. Post-hoc Tukey's test: $* * * * P<0.0001$ when compared with NT, $* P<0.05$ when compared with NT, ${ }^{\#} P<0.0001$ when comparing DA to DA+alec within each time point by post-hoc Sidak's test after two-way ANOVA. (D) HEK cells expressing D2R-FLAG were analyzed by BPA 72 hours after transfection of PKC $\gamma$ or control siRNAs. Labels on blots: total, biotinylated cell surface D2R-FLAG prior to incubation with DA (positive control); strip, biotinylated D2R-FLAG prior to incubation with DA and after stripping biotin from cell surface (negative control); siCtl, transfected with control siRNA pool; siPKC $\gamma$, transfected with siRNA pool targeting PKC $\gamma$. Top blot is a representative streptavidin blot of internalized biotinylated D2R-FLAG, middle blot shows corresponding blot of total cell lysates probed with PKC $\gamma$ antibody, and the bottom blot is the corresponding blot of total cell lysates probed with $\beta$-actin antibody. (E) Graph showing the mean \pm S.D. intensity of D2R-FLAG/ $\beta$-actin from three independent experiments. ${ }^{* *} P<0.001$ when compared with siCtl and siPKC $\gamma,{ }^{\#} P<0.01$ when compared with siCtl+DA. (F) Graph showing the mean \pm S.D. intensity of PKC $\gamma / \beta$-actin from two independent experiments. ${ }^{* * * P}<0.001$ when compared with siCtl and siCtl $+\mathrm{DA}$.

\section{Discussion}

Transactivation of receptor tyrosine kinases by GPCRs in the brain activates signaling cascades that affect diverse processes such as cell proliferation, neurite outgrowth, dendritic spine formation, and neurotransmission. Activation of D2R can transactivate both EGFR and PDGFR to affect cellular processes (Wang et al., 2005; Yoon and Baik, 2013; Shioda et al., 2017). Here, we demonstrated that D2R associates with and activates another receptor tyrosine kinase, ALK, and that ALK promotes D2R internalization. These results suggest that ALK is involved in D2R desensitization, which has implications for the pathophysiology of psychiatric disorders such as addiction and schizophrenia in which $\mathrm{D} 2 \mathrm{R}$ signaling plays a prominent role.

The association of ALK with D2R does not generalize to all DA receptors, because ALK did not associate with D1R, nor did it regulate D1R internalization. D2R and D1R exhibit distinct trafficking mechanisms and fates once internalized (Vickery and von Zastrow, 1999; Bartlett et al., 2005). Whereas D1R is recycled back to the plasma membrane, $\mathrm{D} 2 \mathrm{R}$ is routed to lysosomes and degraded via an association with GPCR-associated sorting protein (GASP) (Bartlett et al., 2005). The association of D2R with ALK represents yet another difference between $\mathrm{D} 1 \mathrm{R}$ and $\mathrm{D} 2 \mathrm{R}$ with respect to trafficking.

Co-IP of ALK and D2R indicates that these proteins interact, probably in a multiprotein complex localized to plasma membrane microdomains such as lipid rafts. Although the coIP results suggest an association between ALK and D2R, this may not be a direct protein-protein interaction. These proteins may instead exist together in a large multiprotein complex at the plasma membrane. The majority of D2R partitions into detergent-resistant structures in cultured cells and in the mouse and rat striatum (Celver et al., 2012; Sharma et al., 2013; Vanderwerf et al., 2015), and treatment of cells with methyl- $\beta$-cyclodextrin, which disrupts lipid rafts, preventing D2R internalization (Cho et al., 2007). In neuroblastoma cells, ALK associates with flotillin, a lipid-raft protein that regulates endocytosis and is localized at the plasma membrane in detergent-resistant structures that act as platforms for cell signaling (Tomiyama et al., 2014; Palacios-Moreno et al., 2015). Treatment with ALK agonist antibodies causes ALK 

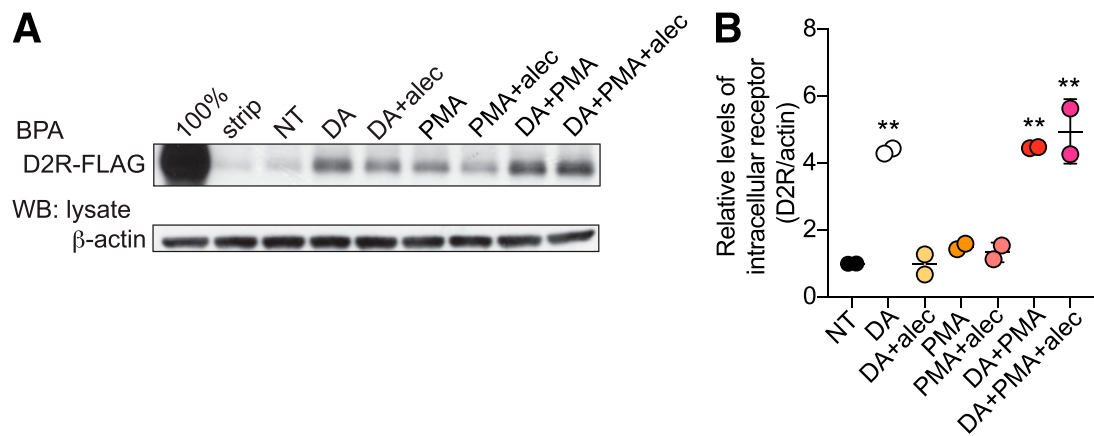

Fig. 5. PKC activation by the phorbol ester PMA abrogates the ability of alectinib to block D2R internalization. (A) HEK cells expressing D2R-FLAG were analyzed by BPA after treatment with various combinations of dopamine (DA, $10 \mu \mathrm{M}$ ), alectinib (alec, $200 \mathrm{nM}$ ), and PMA (10 $\mu \mathrm{M})$. Labels on blots: total, biotinylated cell surface D2R-FLAG prior to incubation with DA (positive control); strip, biotinylated D2R-FLAG prior to incubation with DA and after stripping biotin from cell surface (negative control); NT, no treatment. Top blot is a representative streptavidin blot of internalized biotinylated D2R-FLAG and the bottom blot is the corresponding blot of total cell lysates probed with $\beta$-actin antibody. (B) Graphs showing the mean \pm S.D. intensity of D2R-FLAG/ $\beta$-actin of two independent experiments. ${ }^{*} P<0.01$ by post-hoc Tukey's test after one-way ANOVA when compared with NT, DA + alec, PMA, and PMA+alec.

internalization and degradation via lysosomes (Mazot et al., 2012). A recent study also demonstrated that different isoforms of NUMB, an endocytic adapter protein, differentially direct ALK degradation in lysosomes or its recycling back to the plasma membrane (Wei et al., 2019). Since D2R is also routed to lysosomes and degraded after prolonged DA treatment (Bartlett et al., 2005), it seems probable that both ALK and D2R are eventually trafficked to lysosomes for degradation after prolonged agonist stimulation. More studies are needed to identify if ALK and D2R are colocalized in the same plasma membrane microdomains and endocytic vesicles, and routed to lysosomes after prolonged DA treatment. It is also important to investigate whether flotillin or NUMB are involved in internalization of D2R in an ALK-dependent manner. We attempted to examine subcellular localization of ALK and D2R using immunocytochemistry in HEK and IMR32 cells, but low levels of expression of ALK in HEK cells expressing D2R-FLAG and low levels of endogenous D2R in IMR-32 cells prevented successful confirmation of colocalization of these proteins. An experiment using tagged versions of both ALK and D2R is needed to observe colocalization and their subcellular fates after DA-induced internalization.

One particularly interesting observation from this study is that ALK 140 preferentially associated with D2R over ALK 220 . ALK 140 is a proteolytic product of full length ALK 220 that has been detected in the brain (Iwahara et al., 1997; Lasek et al., 2011). Cleavage of ALK 220 releases an 80-kDa extracellular fragment, the function of which is currently unknown (Moog-Lutz et al., 2005). The remaining ALK 140 contains a portion of the extracellular domain, a transmembrane domain, and an intracellular kinase domain. Why ALK 140 associates with D2R over ALK 220 is not readily apparent. It is possible that a conformational difference in ALK 140 renders it more permissive for association with D2R. Interestingly, DA treatment of IMR-32 cells increased ALK 140 phosphorylation and association of ALK with D2R, providing evidence that activating ALK drives the association with D2R. However, DA treatment of HEK cells expressing D2R-FLAG did not increase ALK and D2R association. The reason for this discrepancy is unknown, but may result from the difference between a heterologous expression system (HEK cells) with high levels of D2R expression and/or the low levels of endogenous ALK expressed in HEK cells resulting in an agonist-independent association. ALK protein levels are very low in HEK cells; however, ALK 140 appears to be more abundant than ALK 220 in these cells (data not shown). Although ALK was able to coimmunoprecipitate with D2R in the absence of DA treatment in HEK cells, this association was not sufficient to drive internalization of D2R. Agonist activation of D2R was required for receptor internalization.

Alectinib treatment of IMR-32 cells decreased levels of pALK 140 even in the absence of DA. Since ALK autophosphorylates, inhibition of ALK kinase activity by alectinib might be expected to decrease basal ALK phosphorylation. ALK 140 has higher basal levels of phosphorylation than ALK 220 , suggesting a different conformation of ALK 140 compared with ALK 220 that might be more permissive for autophosphorylation and inhibition by alectinib. Indeed, it has been reported that truncated ALK lacking the extracellular domain has higher levels of phosphorylation compared with fulllength ALK in cells stimulated with pleiotrophin (PerezPinera et al., 2007). We also previously observed a reduction in basal pALK 140 in IMR-32 cells treated with another ALK inhibitor, NVP-TAE684 (He et al., 2015).

The mechanism(s) by which ALK is activated by D2R is an important area for future research and may be elucidated on the basis of information obtained from other studies on GPCRreceptor tyrosine kinase transactivation. D2R activates the EGFR by increasing the processing of pro-heparin-bound-EGF to active heparin-bound-EGF by matrix metalloproteinases (Wang et al., 2005; Yoon and Baik, 2013). Processing of either full-length ALK itself (to ALK 140) or one of its ligands, such as midkine or FAM150B, could be a potential mechanism (Wellstein, 2012; Reshetnyak et al., 2015). Alternative explanations include the agonist-induced generation of reactive oxygen species that can inactivate protein tyrosine phosphatases that regulate the function of receptor tyrosine kinases, or activation of Src family kinases that may in turn activate receptor tyrosine kinases (Cattaneo et al., 2014).

ALK may regulate the internalization of D2R by PKC activation, because treatment of cells with an inhibitor of the $\mathrm{Ca}^{+}$-dependent PKCs (Gö6976) blocked DA-induced D2R internalization, indicating that a conventional $\mathrm{PKC}$ regulates D2R endocytosis. In addition, DA-induced activation of PLC $\gamma$ and $\mathrm{PKC} \gamma$ were attenuated by alectinib. We also found that treatment with PMA was able to overcome the blockade of 


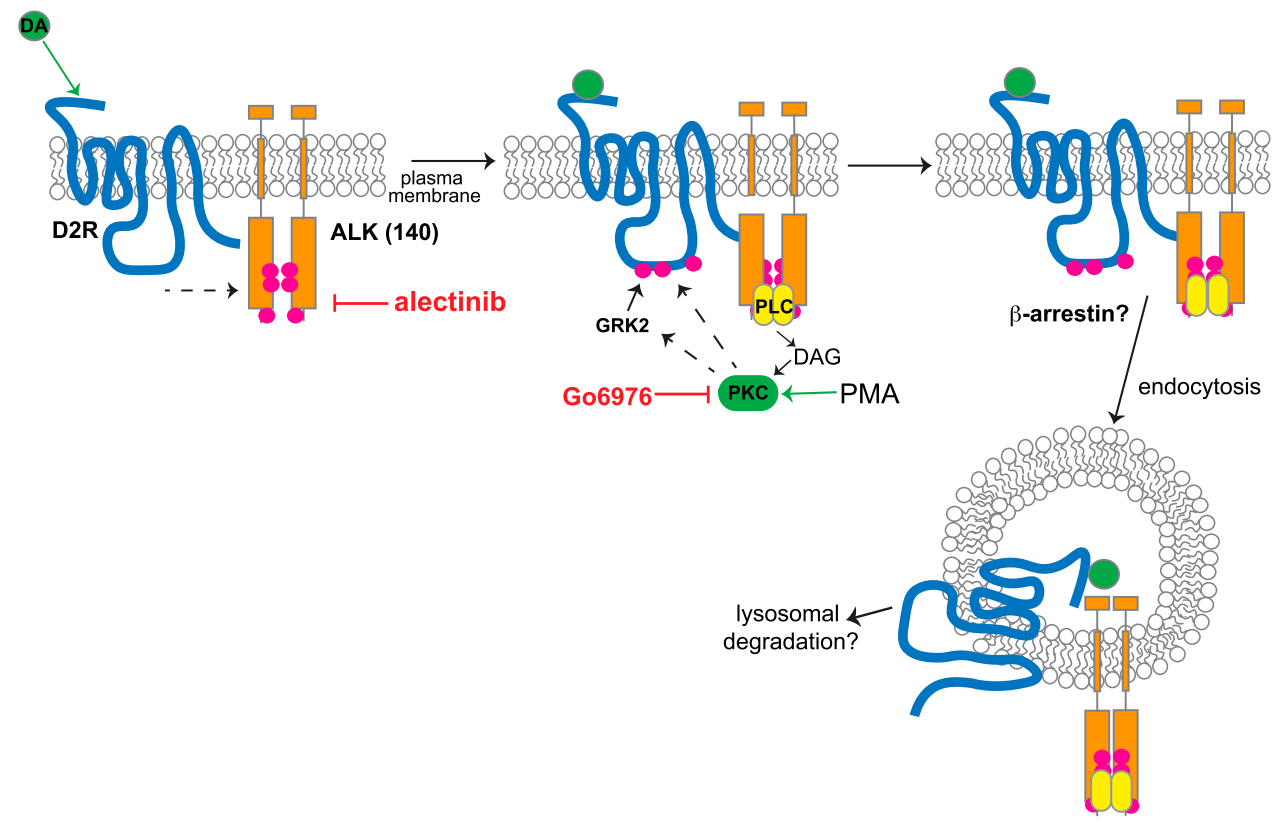

Fig. 6. Proposed model for internalization of D2R mediated by ALK. Dopamine activates D2R, which in turn leads to phosphorylation (pink circles) and activation of ALK 140. Phosphorylated ALK 140 binds to PLC, which is then activated and produces diacylglycerol (DAG). DAG activates $\mathrm{PKC}$. PKC promotes internalization of the D2R either by phosphorylating Ser and Thr residues within the third intracellular loop of D2R, or by phosphorylating $\mathrm{G}$ protein-coupled receptor kinase 2, leading to endocytosis through a $\beta$ arrestin-dependent mechanism and subsequent lysosomal degradation. Blocking activation of ALK by alectinib inhibits internalization of D2R, whereas treatment with the PKC activator PMA eliminates the need for ALK to activate PKC.

D2R internalization by alectinib, suggesting that PKC acts downstream of ALK in regulating D2R endocytosis. PKC is known to be involved in internalization of D2R (Namkung and Sibley, 2004; Thibault et al., 2011; Cho et al., 2013). PMA promotes phosphorylation and internalization of D2R (Namkung and Sibley, 2004), and agonist-induced internalization of D2R is attenuated when putative PKC phosphorylation sites in the third intracellular loop of D2R are mutated (Cho et al., 2010). The PMA treatment conditions used here did not induce a statistically significant internalization of D2R, but this may be owing to a shorter treatment time with PMA (30 minutes) compared with that used by Namkung and Sibley (2 hours) (Namkung and Sibley, 2004). Cho et al. (2007) also did not observe internalization of D2R after 30 minutes of PMA treatment. Combined treatment with PMA and DA was required for $\mathrm{D} 2 \mathrm{R}$ internalization in our experiments. It is possible that the conditions we used induced a subthreshold amount of PKC activation that was increased by DA treatment, possibly through engagement of ALK-independent mechanisms. Another PKC isoform such as $\mathrm{PKC} \beta$ could be involved, because mice treated with a $\operatorname{PKC} \beta$ inhibitor had increased striatal cell surface $\mathrm{D} 2 \mathrm{R}$, suggesting that $\mathrm{PKC} \beta$ is involved in internalization of D2R (Luderman et al., 2015).

Phosphorylation of $\operatorname{PKC} \beta 1, \operatorname{PKC} \gamma$, and $\operatorname{PKC} \theta$ were all increased by DA treatment, but alectinib only decreased pPKC $\gamma$ and $\operatorname{pPKC} \theta$. Since PKC $\theta$ should not be inhibited by Gö6976, we suspected that PKC $\gamma$ may be involved in D2R internalization downstream of ALK. Evidence that PKC $\gamma$ contributes to internalization of $\mathrm{D} 2 \mathrm{R}$ was provided by experiments in which $\mathrm{PKC} \gamma$ expression was attenuated by transfection of siRNAs. A reduction in $\mathrm{PKC} \gamma$ expression resulted in a corresponding decrease in DA-induced internalization of D2R as measured by BPA. Our data provides evidence that activation of $\mathrm{PKC} \gamma$ by ALK may contribute to agonist-induced internalization of $\mathrm{D} 2 \mathrm{R}$.

We propose a model in which DA activation of D2R transactivates ALK by increasing levels of pALK 140, and that ALK, in turn, activates PKC through a PLC-dependent mechanism (Fig. 6). PKC would then either phosphorylate D2R in the third intracellular loop or, alternatively, phosphorylate a GPCR kinase such as GPCR kinase 2 (Chuang et al., 1995; Krasel et al., 2001), which would then phosphorylate D2R and recruit $\beta$-arrestin (Kim et al., 2001), resulting in D2R and ALK endocytosis (Fig. 6). Presumably, D2R endocytosis would lead to receptor desensitization, since we have shown previously that ALK regulates desensitization of D2R in DAergic neurons of the ventral tegmental area (Dutton et al., 2017). ALK inhibitors might therefore represent a novel way to restore normal D2R cell surface expression and signaling in psychiatric disorders in which D2R signaling is attenuated. Further investigation of the regulation of D2R endocytosis by ALK is warranted.

\section{Acknowledgments}

The authors thank Dr. Jennifer L. Whistler for providing the HEK cells expressing flagged-tagged D1R and D2R and protocols for the biotin protection assay. They also thank Drs. Mark Brodie and Amynah Pradhan for helpful comments on this manuscript.

\section{Authorship Contributions \\ Participated in research design: He, Lasek. \\ Conducted experiments: He. \\ Performed data analysis: He, Lasek. \\ Wrote or contributed to writing of the manuscript: Lasek.}

\section{References}

Ariano MA, Sortwell CE, Ray M, Altemus KL, Sibley DR, and Levine MS (1997) Agonist-induced morphologic decrease in cellular D1A dopamine receptor staining. Synapse 27:313-321.

Bartlett SE, Enquist J, Hopf FW, Lee JH, Gladher F, Kharazia V, Waldhoer M, Mailliard WS, Armstrong R, Bonci A, et al. (2005) Dopamine responsiveness is regulated by targeted sorting of D2 receptors. Proc Natl Acad Sci USA 102: $11521-11526$.

Cattaneo F, Guerra G, Parisi M, De Marinis M, Tafuri D, Cinelli M, and Ammendola $\mathrm{R}$ (2014) Cell-surface receptors transactivation mediated by $\mathrm{g}$ protein-coupled receptors. Int J Mol Sci 15:19700-19728.

Celver J, Sharma M, and Kovoor A (2012) D(2)-Dopamine receptors target regulator of G protein signaling 9-2 to detergent-resistant membrane fractions. J Neurochem 120:56-69.

Cho DI, Zheng M, Min C, Kwon KJ, Shin CY, Choi HK, and Kim KM (2013) ARF6 and GASP-1 are post-endocytic sorting proteins selectively involved in the intracellular trafficking of dopamine $\mathrm{D}_{2}$ receptors mediated by GRK and PKC in transfected cells. Br J Pharmacol 168:1355-1374. 
Cho D, Zheng M, Min C, Ma L, Kurose H, Park JH, and Kim KM (2010) Agonistinduced endocytosis and receptor phosphorylation mediate resensitization of dopamine D(2) receptors. Mol Endocrinol 24:574-586.

Cho EY, Cho DI, Park JH, Kurose H, Caron MG, and Kim KM (2007) Roles of protein kinase $\mathrm{C}$ and actin-binding protein 280 in the regulation of intracellular trafficking of dopamine D3 receptor. Mol Endocrinol 21:2242-2254.

Chuang TT, LeVine H III, and De Blasi A (1995) Phosphorylation and activation of beta-adrenergic receptor kinase by protein kinase C. J Biol Chem 270: 18660-18665.

Crockett DK, Lin Z, Elenitoba-Johnson KS, and Lim MS (2004) Identification of NPM-ALK interacting proteins by tandem mass spectrometry. Oncogene 23: $2617-2629$

Dutton JW III, Chen H, You C, Brodie MS, and Lasek AW (2017) Anaplastic lymphoma kinase regulates binge-like drinking and dopamine receptor sensitivity in the ventral tegmental area. Addict Biol 22:665-678.

Grady EF, Böhm SK, and Bunnett NW (1997) Turning off the signal: mechanisms that attenuate signaling by $\mathrm{G}$ protein-coupled receptors. Am $J$ Physiol 273: G586-G601.

He D, Chen H, Muramatsu H, and Lasek AW (2015) Ethanol activates midkine and anaplastic lymphoma kinase signaling in neuroblastoma cells and in the brain $J$ Neurochem 135:508-521.

Howes OD, McCutcheon R, Owen MJ, and Murray RM (2017) The role of genes, stress, and dopamine in the development of schizophrenia. Biol Psychiatry $\mathbf{8 1}$ $9-20$

Iwahara T, Fujimoto J, Wen D, Cupples R, Bucay N, Arakawa T, Mori S, Ratzkin B, and Yamamoto T (1997) Molecular characterization of ALK, a receptor tyrosine kinase expressed specifically in the nervous system. Oncogene 14:439-449.

Janoueix-Lerosey I, Lequin D, Brugières L, Ribeiro A, de Pontual L, Combaret V, Raynal V, Puisieux A, Schleiermacher G, Pierron G, et al. (2008) Somatic and germline activating mutations of the ALK kinase receptor in neuroblastoma. $\mathrm{Na}$ ture 455:967-970.

Kim KM, Valenzano KJ, Robinson SR, Yao WD, Barak LS, and Caron MG (2001) Differential regulation of the dopamine D2 and D3 receptors by G protein-coupled receptor kinases and beta-arrestins. J Biol Chem 276:37409-37414.

Kim SJ, Kim MY, Lee EJ, Ahn YS, and Baik JH (2004) Distinct regulation of internalization and mitogen-activated protein kinase activation by two isoforms of the dopamine D2 receptor. Mol Endocrinol 18:640-652.

Kotecha SA, Oak JN, Jackson MF, Perez Y, Orser BA, Van Tol HH, and MacDonald JF (2002) A D2 class dopamine receptor transactivates a receptor tyrosine kinase to inhibit NMDA receptor transmission. Neuron 35:1111-1122.

Krasel C, Dammeier S, Winstel R, Brockmann J, Mischak H, and Lohse MJ (2001) Phosphorylation of GRK2 by protein kinase $\mathrm{C}$ abolishes its inhibition by calmodulin. J Biol Chem 276:1911-1915.

Lacey MG, Mercuri NB, and North RA (1987) Dopamine acts on D2 receptors to increase potassium conductance in neurones of the rat substantia nigra zona compacta. J Physiol 392:397-416.

Lasek AW, Lim J, Kliethermes CL, Berger KH, Joslyn G, Brush G, Xue L, Robertson M, Moore MS, Vranizan K, et al. (2011) An evolutionary conserved role for anaplastic lymphoma kinase in behavioral responses to ethanol. PLoS One 6:e22636.

Luderman KD, Chen R, Ferris MJ, Jones SR, and Gnegy ME (2015) Protein kinase C beta regulates the $\mathrm{D}_{2}$-like dopamine autoreceptor. Neuropharmacology $\mathbf{8 9}$ 335-341.

Mazot P, Cazes A, Dingli F, Degoutin J, Irinopoulou T, Boutterin MC, Lombard B Loew D, Hallberg B, Palmer RH, et al. (2012) Internalization and down-regulation of the ALK receptor in neuroblastoma cell lines upon monoclonal antibodies treatment. PLoS One 7:e33581.

Moog-Lutz C, Degoutin J, Gouzi JY, Frobert Y, Brunet-de Carvalho N, Bureau J, Créminon C, and Vigny M (2005) Activation and inhibition of anaplastic lymphoma kinase receptor tyrosine kinase by monoclonal antibodies and absence of agonist activity of pleiotrophin. J Biol Chem 280:26039-26048.

Morris SJ, Van-Ham II, Daigle M, Robillard L, Sajedi N, and Albert PR (2007) Differential desensitization of dopamine D2 receptor isoforms by protein kinase C: the importance of receptor phosphorylation and pseudosubstrate sites. Eur $J$ Pharmacol 577:44-53.

Namkung Y and Sibley DR (2004) Protein kinase C mediates phosphorylation, desensitization, and trafficking of the D2 dopamine receptor. J Biol Chem $\mathbf{2 7 9}$ 49533-49541.

Nimitvilai S, Arora DS, and Brodie MS (2012) Reversal of dopamine inhibition of dopaminergic neurons of the ventral tegmental area is mediated by protein kinase C. Neuropsychopharmacology 37:543-556.
Nimitvilai S and Brodie MS (2010) Reversal of prolonged dopamine inhibition of dopaminergic neurons of the ventral tegmental area. J Pharmacol Exp Ther 333: $555-563$.

Oak JN, Lavine N, and Van Tol HH (2001) Dopamine D(4) and D(2L) Receptor Stimulation of the Mitogen-Activated Protein Kinase Pathway Is Dependent on trans-Activation of the Platelet-Derived Growth Factor Receptor. Mol Pharmacol 60:92-103.

Palacios-Moreno J, Foltz L, Guo A, Stokes MP, Kuehn ED, George L, Comb M, and Grimes ML (2015) Neuroblastoma tyrosine kinase signaling networks involve FYN and LYN in endosomes and lipid rafts. PLOS Comput Biol 11:e1004130.

Palmer RH, Vernersson E, Grabbe C, and Hallberg B (2009) Anaplastic lymphoma kinase: signalling in development and disease. Biochem J 420:345-361.

Perez-Pinera P, Zhang W, Chang Y, Vega JA, and Deuel TF (2007) Anaplastic lymphoma kinase is activated through the pleiotrophin/receptor protein-tyrosine phosphatase beta/zeta signaling pathway: an alternative mechanism of receptor tyrosine kinase activation. J Biol Chem 282:28683-28690.

Reshetnyak AV, Murray PB, Shi X, Mo ES, Mohanty J, Tome F, Bai H, Gunel M, Lax I, and Schlessinger J (2015) Augmentor $\alpha$ and $\beta$ (FAM150) are ligands of the receptor tyrosine kinases ALK and LTK: hierarchy and specificity of ligandreceptor interactions. Proc Natl Acad Sci USA 112:15862-15867.

Sattu K, Hochgräfe F, Wu J, Umapathy G, Schönherr C, Ruuth K, Chand D, Witek B, Fuchs J, Li PK, et al. (2013) Phosphoproteomic analysis of anaplastic lymphoma kinase (ALK) downstream signaling pathways identifies signal transducer and activator of transcription 3 as a functional target of activated ALK in neuroblastoma cells. FEBS J 280:5269-5282.

Sharma M, Celver J, Octeau JC, and Kovoor A (2013) Plasma membrane compartmentalization of D2 dopamine receptors. J Biol Chem 288:12554-12568.

Shioda N, Yabuki Y, Wang Y, Uchigashima M, Hikida T, Sasaoka T, Mori H, Watanabe M, Sasahara M, and Fukunaga K (2017) Endocytosis following dopamine $\mathrm{D}_{2}$ receptor activation is critical for neuronal activity and dendritic spine formation via Rabex-5/PDGFR $\beta$ signaling in striatopallidal medium spiny neurons. Mol Psychiatry 22:1205-1222.

Thibault D, Albert PR, Pineyro G, and Trudeau LE (2011) Neurotensin triggers dopamine D2 receptor desensitization through a protein kinase C and betaarrestin1-dependent mechanism. J Biol Chem 286:9174-9184.

Tomiyama A, Uekita T, Kamata R, Sasaki K, Takita J, Ohira M, Nakagawara A, Kitanaka C, Mori K, Yamaguchi H, et al. (2014) Flotillin-1 regulates oncogenic signaling in neuroblastoma cells by regulating ALK membrane association. Cancer Res 74:3790-3801.

Van den Eynden J, Umapathy G, Ashouri A, Cervantes-Madrid D, Szydzik J, Ruuth K, Koster J, Larsson E, Guan J, Palmer RH, et al. (2018) Phosphoproteome and gene expression profiling of ALK inhibition in neuroblastoma cell lines reveals conserved oncogenic pathways. Sci Signal 11 DOI: 10.1126/scisignal.aar5680.

Vanderwerf SM, Buck DC, Wilmarth PA, Sears LM, David LL, Morton DB, and Neve KA (2015) Role for Rab10 in methamphetamine-induced behavior. PLoS One 10: e0136167.

Vickery RG and von Zastrow M (1999) Distinct dynamin-dependent and -independent mechanisms target structurally homologous dopamine receptors to different endocytic membranes. J Cell Biol 144:31-43.

Volkow ND and Morales M (2015) The brain on drugs: from reward to addiction. Cell 162:712-725

Wang C, Buck DC, Yang R, Macey TA, and Neve KA (2005) Dopamine D2 receptor stimulation of mitogen-activated protein kinases mediated by cell type-dependent transactivation of receptor tyrosine kinases. J Neurochem 93:899-909.

Wei R, Liu X, Voss C, Qin W, Dagnino L, Li L, Vigny M, and Li SS (2019) NUMB regulates the endocytosis and activity of the anaplastic lymphoma kinase in an isoform-specific manner. $J$ Mol Cell Biol DOI: 10.1093/jmcb/mjz003 [published ahead of print].

Wellstein A (2012) ALK receptor activation, ligands and therapeutic targeting in glioblastoma and in other cancers. Front Oncol 2:192.

Yoon S and Baik JH (2013) Dopamine D2 receptor-mediated epidermal growth factor receptor transactivation through a disintegrin and metalloprotease regulates dopaminergic neuron development via extracellular signal-related kinase activation. J Biol Chem 288:28435-28446.

Address correspondence to: Dr. Amy W. Lasek, Department of Psychiatry, University of Illinois at Chicago, 1601 West Taylor Street, MC 912, Chicago, IL 60612. E-mail: alasek@uic.edu 